\title{
Reducing the Dosing Frequency of Selective Digestive Tract Decontamination to Three Times Daily Provides Effective Decontamination of Potentially Pathogenic Micro-Organisms
}

Jara Rebekka de la Court ( $\nabla$ j.delacourt@amsterdamumc.nl )

Amsterdam UMC - locatie VUmc

Kim C.E. Sigaloff

Amsterdam UMC - Locatie VUMC: Amsterdam UMC Locatie VUmc

Thomas Groot

Amsterdam UMC - Locatie VUMC: Amsterdam UMC Locatie VUmc

Johan I. van der Spoel

Amsterdam UMC - Locatie VUMC: Amsterdam UMC Locatie VUmc

Rogier P. Schade

Amsterdam UMC - Locatie VUMC: Amsterdam UMC Locatie VUmc

\section{Research Article}

Keywords: Selective decontamination, antibacterial agents, antimicrobial stewardship, infection prevention, intensive care unit

Posted Date: February 12th, 2021

DOl: https://doi.org/10.21203/rs.3.rs-186916/v1

License: (c) (i) This work is licensed under a Creative Commons Attribution 4.0 International License.

Read Full License

Version of Record: A version of this preprint was published at European Journal of Clinical Microbiology \& Infectious Diseases on April 1st, 2021. See the published version at https://doi.org/10.1007/s10096021-04234-1. 


\section{Abstract}

\section{Purpose}

This study evaluated the effectiveness of selective digestive tract decontamination (SDD) application three times daily (t.i.d.) compared to the standard four times daily (q.i.d.).

\section{Methods}

Retrospective equivalence study with a before-and-after design on a tertiary ICU in which the SDD frequency was reduced from q.i.d. to t.i.d. All patients with ICU admissions $\geq 72 h$, and with $\geq 2$ surveillance cultures collected on different dates were included in this study. We compared successful decontamination of Gram-negative bacteria (GNB). Furthermore, time to decontamination, ICU-acquired GNB bacteraemia and 28-day mortality were compared between the two groups.

\section{Results}

In total 1958 ICU admissions (1236 q.i.d., 722 t.i.d). Decontamination was achieved during the first week of admission in $77 \%$ and $76 \%$ of patients receiving SDD q.i.d and t.i.d., respectively. Successful decontamination within 14 days (without consecutive acquisition of Gram-negative bacteria) was achieved in $69.3 . \%$ of the admissions with q.i.d. versus $66.8 \%$ in t.i.d. SDD ( $p$-value $=0.2519$ ). The proportions of successful decontamination of GNB were equivalent in both groups $(-0.025,98 \% \mathrm{Cl}$ : -0.087 ; 0.037). There was no significant difference in time to decontamination between the two regimens (logrank test p-value $=0.55$ ). Incidence (episodes $/ 1000$ days) of ICU-acquired GNB bacteraemia was 0.9 in both groups and OR for death at day 28 in the t.i.d. group compared to the q.i.d. group was 0.99 (95\% confidence interval, 0.80-1.21).

\section{Conclusions}

This study shows that a t.i.d. application regimen provides equally effective SDD compared to the standard q.i.d. regime, for both microbiological and clinical outcome measures.

\section{Introduction}

Infection is a major complication among patients in the intensive care unit (ICU) resulting in additional morbidity, higher risk of mortality and increasing health care costs.[1] Selective digestive tract decontamination (SDD) is a common measure to prevent infections on the ICU. The principle behind SDD is that by reducing the numbers of potentially pathogenic microorganisms (PPM) in the gut, the risk of ICU-acquired infections can be reduced.[2] Intestinal decontamination of Gram-negative bacteria (GNB) was associated with a three-fold reduction in ICU-acquired bacteraemia with GNB.[3] The positive effect of SDD on clinical outcomes, i.e. Improved survival and less infectious complications while maintaining a low prevalence of antibiotic resistance, has been demonstrated by three cluster-randomized studies. [3-5] 
The SDD regime is applied in ventilated patients with an expected duration of artificial ventilation $>48$ hours and consists of a mixture of non-absorbable antimicrobials combined with intravenous cefotaxime during the first four days of ICU admission. SDD is applied q.i.d. and this frequency has remained unchanged since the first SDD studies in the 1980s. There have been no studies that evaluated the optimal dosing frequency of SDD in order to achieve GNB decontamination. Reducing the dosing frequency of SDD paste application from four to three times daily (t.i.d.) would lower antimicrobial consumption and related health care costs. More importantly, by reducing the dosing frequency the nightly administration of SDD paste application could be omitted, thereby preventing sleep interruption. Facilitating uninterrupted sleep reduces the incidence of delirium and is essential for adequate immune, metabolic, and endocrine functioning.[6, 7] In an attempt to increase the quality of sleep for ICU patients, the dosing frequency of SDD application was reduced from q.i.d. to t.i.d in 2017. This t.i.d. SDD dosing regimen has been the standard of care since. This provided us with the opportunity to perform a beforeand after study comparing the efficacy q.i.d. versus the t.i.d. SDD application frequency, in terms of GNB decontamination of the digestive tract and subsequent ICU-acquired GNB-infections.

\section{Materials And Methods}

\section{Setting, Design and Population}

We retrospectively studied electronic microbiology and patient data gathered in the period from November 2011 until July 2019 on the ICU of the Amsterdam University Medical Centre, location VU medical centre (VUmc), a 28 bed ICU in a 730-bed tertiary care centre in the Netherlands. Data were derived from an automated database combining laboratory data with pseudo-anonymous patient data from the Electronic patient dossier (EPD). This database was constructed for antimicrobial stewardship and infection prevention purposes and consists of microbiological data, admission and discharge data, Medicine Administration Records, Surgical interventions and a small amount of patient data: sex, date of birth, date of death. Data visualization was performed using TIBCO ${ }^{\circledR}$ Spotfire ${ }^{\circledR}$. The non-absorbable antibiotics in the SDD regimen consist of application of paste (colistin $2 \%$, tobramycin $2 \%$, amphoterin B $2 \%$ ) in the oral cavity and of suspension (colistin $100 \mathrm{mg}$, tobramycin $80 \mathrm{mg}$, amphotericin $500 \mathrm{mg}$ ) via the nasogastric tube. This regimen was applied q.i.d. until 26-05-2017, thereafter the same regimen was applied t.i.d. Patients admitted to the ICU also receive 4 days of intravenous cefotaxime q.i.d. $1 \mathrm{~g}$, this practice did not change over the course of time.

All adult patients with an ICU admission of at least $72 \mathrm{~h}$ and with at least 2 surveillance cultures drawn on two separate days were included in the analysis.

\section{Microbiological Methods}

Surveillance cultures were taken on admission to the ICU and thereafter once a week on Mondays for pharynx and anus, and on Mondays and Thursdays for sputum. All surveillance cultures were included in 
the analysis. Surveillance cultures within $72 \mathrm{~h}$ of admission represented the flora at admission on the ICU and are further called baseline surveillance cultures.

Based on previous literature the following aerobic GNB were defined as PPMs: Klebsiella, Enterobacter, Citrobacter, Proteus, Morganella, Serratia, Acinetobacter and Pseudomonas species.[8] By assessing the prevalence of GNB in the blood cultures of the patients in our cohort we found that Stenotrophomonas maltophilia was also a frequent cultured pathogen in the study period and was therefore added as an PPM. Because we also want to assess the reduction of carriage of endogenous "normal" but potentially pathogenic flora we added Escherichia Coli to the list.

In the Amsterdam UMC medical microbiology laboratories, antimicrobial susceptibility was tested using automated systems, gradient tests and/or using the disk diffusion method.

Decontamination was defined as the reduction of Gram-negative bacterial load to a level at which surveillance cultures are negative (rectal or faeces, pharyngeal and sputum). The number of days in which decontamination should occur to be considered successful, i.e. adequate to reduce infectious complications and mortality, has not been previously defined. In the study of de Smet et al -- in which the relationship between SDD and reduction of mortality was confirmed -- the frequency of GNB isolation from rectal swabs among patients receiving SDD was reduced from $56 \%$ at day 3 to $15 \%$ at day 14.[4] The SDD regimen used in the study of de Smet et al was identical to the q.i.d. regimen used in this study. Therefore we chose to define successful decontamination as a surveillance culture result negative for GNB within 14 days without positive follow-up surveillance culture for GNB during ICU-admission, i.e. follow-up lasted until the moment of discharge from the ICU.

In case of new fever two blood cultures were drawn. ICU-acquired bacteremia with GNB was defined as bacteraemia occurring at least 48 hours after ICU admission with growth of either Enterobacterales or glucose-nonfermenting Gram-negative rods, without documented bacteraemia with the same species in the first 48 hours of ICU admission. Polymicrobial bacteraemia was defined when one or more microorganisms were isolated from one or more blood cultures, and clinical evidence suggested they had arisen from a common source and were part of the same episode. If the source was unknown, all positive blood cultures occurring within 48 hours of each other are considered as a single bacteraemia.

28-day all-cause mortality is defined as death for any cause within 28 days after the date of admission to the ICU.

Due to the before-after study design, we anticipated that time dependent factors such as antimicrobial resistance could introduce bias. We described the combined prevalence of susceptibility for the components of SDD (tobramycin and colistin) in surveillance cultures at baseline.

\section{Analysis}


Two groups were formed on the basis of the dosing frequency of SDD, q.i.d. versus t.i.d. Continuous variables are presented as median and interquartile range. Categorical variables are presented as percentages. For the difference in proportion of patients with successful decontamination of PPMs in both groups and for comparison of susceptibility of Gram-negative bacteria at baseline a Chi-square test was used. For the difference in time to decontamination of PPMs from surveillance cultures a Kaplan Meier curve was used. For equivalence testing the two one-sided test (TOST) procedure was used. The largest clinically acceptable effect for which equivalence can be declared was a mean difference of $10 \%$. The equivalence limit was set to $0.1\left(d_{E}=0.1\right)$. All data available was used, no formal sample size was calculated. Odds ratio was calculated to compare 28-day all-cause mortality between the two groups. Data analysis was performed using R Statistical Software (version 3.6.1; R Foundation for Statistical Computing, Vienna, Austria).

\section{Results}

Data was gathered from 3943 admissions, in 3662 ICU patients. A total of 1958 admissions in 1851 patients met the criteria for inclusion in our study. The q.i.d. cohort consisted of 1236 admissions, the t.i.d. cohort consisted of 722 admissions. Baseline characteristics are shown in table 1. The cohorts were comparable with regard to duration of admission, age at admission and percentage of male. Figure 1 shows, for both cohorts, the percentage of patients with positive cultures at the different time points during the admission. In both cohorts, successful decontamination was primarily initiated during the first week of admission, with 77-76\% (q.i.d - t.i.d.) of patients with GNB positive cultures at admission to 22$24 \%$ (q.i.d - t.i.d.) after one week. The percentage of patients with GNB positive cultures varied between $17-24 \%$ during 6-14 days. However after 15 days, rate of GNB positive cultures increased to $28 \%$ in both groups. Table 2 shows the proportion of successful decontamination in the two groups. Successful decontamination, defined as GNB negative surveillance cultures within 14 days without any further GNB detection in surveillance cultures thereafter, was not significantly different between the two groups (Table 2). To show non-inferiority of the t.i.d. regime, equivalence test of the proportions of successful decontamination was performed (Figure 2). With an equivalence bound of 0.1 and a $98 \%$ confidence interval, the proportions of successful decontamination of GNB are equivalent in both groups. The time to decontamination of GNB is shown in figure 3. The log-rank test, to compare time to decontamination of GNB between the two cohorts, did not show any difference between the groups ( $p$-value of 0.55 ).

We observed 27 episodes of ICU-acquired bacteraemia with GNB during the study period, 17/1236 before $(1.4 \%)$ and 10/722 (1.4\%) after adjustment of SDD application frequency, with an incidence of 0.9 episodes/1000 ICU days in both. Causative pathogens in intensive care unit-acquired bacteraemia are shown in table 3. 28 -day all-cause mortality was $26.1 \%$ and $25.6 \%$ in the q.i.d. and t.i.d. groups, odds ratios for death at day 28 in the t.i.d. group compared to the q.i.d. group was 0.99 ( $95 \%$ confidence interval [Cl], 0.80-1.21).

To control for potential changes in resistance epidemiology between the two time periods, especially with regard to the incidence of bacteria that were susceptible to the SDD antibiotics, we compared the 
surveillance cultures at baseline (Table 4). Between $72-71 \%$ (for the q.i.d. and t.i.d. group) of all admissions started with GNB positive surveillance cultures at baseline. Susceptibility for tobramycin or colistin in Gram-negative bacteria in baseline surveillance cultures was $97.3 \%$ and $96.8 \%$ (for the q.i.d. and t.i.d. group) with a p-value of 0.61 using the chi-square test. We conclude that the baseline epidemiology at admission on the ICU is comparable, and that our results are not biased by an epidemiological shift in susceptibility rate between the two historical cohorts.

The incidence of VAP on our ward has been shown to be 3.3/1,000 ventilation days.[9] Prevalence measurements for the national 'PREZIES' survey of hospital infections are performed every three months. $[10,11]$ Based on these surveys, the prevalence of VAP in q.i.d. cohort (median $0.1 \%$ of admitted patients, range $0-19 \%$ ) did not significant increase after change to the t.i.d. regime (median $0.1 \%$ of admitted patients, range $0-21 \%$ ).

\section{Discussion}

Despite its common use, the optimal SDD dosing regime has not previously been evaluated in a clinical setting. The present study demonstrated that a t.i.d. application regime application regimen provides equally effective selective digestive tract decontamination compared to the standard q.i.d. regime. SDD effectiveness was demonstrated within a large patient population $(n=1958)$ receiving either t.i.d. $(n=$ 722) or q.i.d. $(n=1236)$ administration. Several outcome measures support our conclusion. First, the proportion of successful decontamination was equal in both groups. Second, the time to decontamination of GNB did not, at any time point, differ significantly. Finally, we found no significant differences in clinically relevant outcomes (i.e. ICU-acquired bacteraemia and 28-day all-cause mortality) between the two cohorts.

Although the goal of SDD is digestive tract decontamination and subsequent reduction of ICU-acquired infection, the four cluster-randomized controlled trials that have previously investigated the efficacy of SDD did not report the time to- or success of decontamination.[3-5, 12] The primary outcome of our study can therefore not directly be compared to these trials. However, the close association between gut (de-)colonization and ICU-acquired infection is well established.[13-18] Frencken et al showed that both rectal and respiratory tract colonization were associated with bacteraemia (cause-specific hazard ratios, 7.37 [95\% Cl, 3.25-16.68] and 2.56 [95\% Cl, 1.09-6.03], respectively).[14] Oostdijk et al found that respiratory tract decolonization and intestinal tract decolonization was associated with a $33 \%$ and $45 \%$ reduction in the occurrence of intensive care unit acquired Gram-negative bacteraemia, respectively. Moreover, Oostdijk et al reported a reduction of proportion of colonization in patients treated with SDD throughout intensive care unit stay from approximately $30 \%$ at day 1 to $15-20 \%$ at day 20.[13] The fact that decontamination rates found in our study are comparable to the results found in the large prospective study of the Smet et al (i.e. $85 \%$ of patients cultured after 14 days are decolonized from GNB), in which clinical effectiveness of SDD application was proven, is a clear indication that t.i.d. administration of SDD is clinically effective and safe. $[4,13]$ Furthermore, the secondary clinical outcomes 
defined in our study (ICU-acquired bacteraemia and 28-day all-cause mortality), in which t.i.d. proved to be non-inferior to q.i.d, supports this conclusion.

Strengths of our study are the size of our study population and the detailed information about intestinal colonization during SDD. This study provides, for the first time, detailed insight into the underlying dynamics of culture-results during SDD. We demonstrated equal microbiological and clinical effectiveness of less frequent dosing. This is reflected in a stable incidence of ICU-acquired GNB bacteraemia and 28-day all-cause mortality. Our study also has limitations. We used a monocentric retrospective approach, using a historical control group. We had no detailed clinical information on ventilator associated pneumonias in individual patients. On a population level, however, we noted no significant change in prevalence of VAP since the introduction of the t.i.d. application regime.[11] Besides, Bergmans et al showed previously that decolonization of the respiratory tract results in a relative risk reduction of $67 \%$ in the incidence of VAP. This makes a difference in VAP incidence despite the equivalent decontamination rates in our cohorts unlikely.[19] The retrospective design precludes correction for hidden variables in the original data that might have confounded the results. Yet, potential confounders in the original uncontrolled data are likely to be present in both groups (t.i.d. versus q.i.d.). Specifically, we ruled out an epidemiological shift in susceptibility for the SDD antibiotics, which could otherwise have biased the results.

Our findings support a t.i.d. SDD application frequency in the ICU. This new regime was designed as an sleep-promoting intervention on the ICU. Many sleep-disturbing factors are present on the ICU, but clinical interventions are one of the most important disruptive factors and should therefore be avoided.[20] Furthermore any unnecessary antibiotic use should be avoided to reduce the harm that can result from antibiotic-associated adverse events.[21] During the t.i.d. SDD application period of three years tobramycin and colistin resistance did not change, which is in line with previous studies assessing antibiotic resistance during the use of SDD.[22-24]

\section{Conclusion}

Based on time to- and success of decontamination of Gram-negative bacteria, incidence of ICU-acquired GNB bacteraemia (0.9/1000 ICU days) and 28-day all-cause mortality there is no difference between a t.i.d. and a q.i.d. SDD application regime. These study findings justify implementation of a t.i.d. SDD application regimen in ICUs where a standard (q.i.d.) regimen is in place.

\section{Declarations}

\section{Funding:}

No financial support was used for this study.

\section{Conflicts of interest/Competing interests:}


The authors have no conflicts of interest to declare that are relevant to the content of this article.

\section{Availability of data and material:}

Data is available upon request

\section{Code availability:}

Code is available upon request

\section{Authors' contributions:}

All authors contributed to the study conception and design. Data collection were performed by Thomas Groot and Jara R. de la Court, data analysis was performed by Jara R. de la Court. The first draft of the manuscript was written by Jara R. de la Court and all authors commented on earlier versions of the manuscript. All authors read and approved the final manuscript.

\section{Ethics approval:}

This study was approved by the Medical Ethical Committee of the VUmc.

\section{Consent to participate:}

Informed consent was not required for this retrospective study for which pseudo-anonymous data was collected and analysed, in this manuscript data is presented anonymized.

\section{Acknowledgments}

We thank the ICU, medical microbiology laboratory and the microbiology data-team of the Amsterdam UMC.

\section{References}

1. Vincent JL: Nosocomial infections in adult intensive-care units. Lancet 2003; 361(9374):2068-2077

2. Voort P, van Saene H: Selective Digestive Tract Decontamination in Intensive Care Medicine: a Practical Guide to Controlling Infection; Springer, 2008

3. de Jonge E, Schultz MJ, Spanjaard L, Bossuyt PM, Vroom MB, Dankert J, Kesecioglu J: Effects of selective decontamination of digestive tract on mortality and acquisition of resistant bacteria in intensive care: a randomised controlled trial. Lancet 2003, 362(9389):1011-1016. 
4. de Smet AM, Kluytmans JA, Cooper BS, Mascini EM, Benus RF, van der Werf TS, van der Hoeven JG, Pickkers P, Bogaers-Hofman D, van der Meer NJ et al: Decontamination of the digestive tract and oropharynx in ICU patients. The New England journal of medicine 2009, 360(1):20-31.

5. Oostdijk EAN, Kesecioglu J, Schultz MJ, Visser CE, de Jonge E, van Essen EHR, Bernards AT, Purmer I, Brimicombe R, Bergmans D et al: Effects of Decontamination of the Oropharynx and Intestinal Tract on Antibiotic Resistance in ICUs: A Randomized Clinical Trial. JAMA 2014, 312(14):1429-1437.

6. Gamaldo CE, Shaikh AK, McArthur JC: The sleep-immunity relationship. Neurologic clinics 2012, 30(4):1313-1343.

7. Figueroa-Ramos MI, Arroyo-Novoa CM, Lee KA, Padilla G, Puntillo KA: Sleep and delirium in ICU patients: a review of mechanisms and manifestations. Intensive care medicine 2009, 35(5):781-795.

8. an Saene HK, Damjanovic V, Murray AE, de la Cal MA: How to classify infections in intensive care units--the carrier state, a criterion whose time has come? The Journal of hospital infection 1996, 33(1):1-12.

9. Kaiser AM, de Jong E, Evelein-Brugman SFM, Peppink JM, Vandenbroucke-Grauls CMJE, Girbes ARJ: Development of trigger-based semi-automated surveillance of ventilator-associated pneumonia and central line-associated bloodstream infections in a Dutch intensive care Annals of Intensive Care 2014, 4:40

10. van der Kooi TII, Boshuizen H, Wille JC, de Greeff SC, van Dissel JT, Schoffelen AF, van Gaalen RD: Using flexible methods to determine risk factors for ventilator-associated pneumonia in the Netherlands. PloS one 2019, 14(6):e0218372.

11. RIVM: annual figures 2019: Academic hospitals PREZIES - version: juli 2020. Documentversion: 1.0. Available at: https://www.rivm.nl/documenten/prezies-po-jaarcijfers-2019-academischeziekenhuizen. Accessed September 9, 2020

12. Wittekamp BH, Plantinga NL, Cooper BS, Lopez-Contreras J, Coll P, Mancebo J, Wise MP, Morgan MPG, Depuydt P, Boelens J et al: Decontamination Strategies and Bloodstream Infections With Antibiotic-Resistant Microorganisms in Ventilated Patients: A Randomized Clinical Trial. Jama 2018, 320(20):2087-2098.

13. Oostdijk EA, de Smet AM, Kesecioglu J, Bonten MJ: The role of intestinal colonization with gramnegative bacteria as a source for intensive care unit-acquired bacteremia. Critical care medicine 2011, 39(5):961-966.

14. Frencken JF, Wittekamp BHJ, Plantinga NL, Spitoni C, van de Groep K, Cremer OL, Bonten MJM: Associations Between Enteral Colonization With Gram-Negative Bacteria and Intensive Care UnitAcquired Infections and Colonization of the Respiratory Tract. Clin Infect Dis 2018, 66(4):497-503.

15. Freedberg DE, Zhou MJ, Cohen ME, Annavajhala MK, Khan S, Moscoso DI, Brooks C, Whittier S, Chong $\mathrm{DH}$, Uhlemann A-C et al: Pathogen colonization of the gastrointestinal microbiome at intensive care unit admission and risk for subsequent death or infection. Intensive care medicine 2018, 44(8):1203-1211. 
16. Gomez-Zorrilla S, Camoez M, Tubau F, Canizares R, Periche E, Dominguez MA, Ariza J, Pena C: Prospective observational study of prior rectal colonization status as a predictor for subsequent development of Pseudomonas aeruginosa clinical infections. Antimicrob Agents Chemother 2015, 59(9):5213-5219.

17. Gorrie CL, Mirceta M, Wick RR, Edwards DJ, Thomson NR, Strugnell RA, Pratt NF, Garlick JS, Watson KM, Pilcher DV et al: Gastrointestinal Carriage Is a Major Reservoir of Klebsiella pneumoniae Infection in Intensive Care Patients. Clin Infect Dis 2017, 65(2):208-215.

18. Latibeaudiere R, Rosa R, Laowansiri P, Arheart K, Namias N, Munoz-Price LS: Surveillance cultures growing carbapenem-Resistant Acinetobacter baumannii predict the development of clinical infections: a retrospective cohort study. Clin Infect Dis 2015, 60(3):415-422.

19. Bergmans,DC, Bonten MJ, Gaillard CA, Paling JC, van der Geest S, van Tiel FH, Beysen AJ, de Leeuw PW, Stobberingh EE: Prevention of ventilator-associated pneumonia by oral decontamination: a prospective, randomized, double-blind, placebo-controlled study. Am J Respir Crit Care Med 2001, 164:382-8

20. Alsulami G, Rice AM, Kidd L: Prospective repeated assessment of self-reported sleep quality and sleep disruptive factors in the intensive care unit: acceptability of daily assessment of sleep quality. BMJ open 2019, 9(6):e029957.

21. Tamma PD, Avdic E, Li DX, Dzintars K, Cosgrove SE: Association of Adverse Events With Antibiotic Use in Hospitalized Patients. JAMA internal medicine 2017, 177(9):1308-1315.

22. Buitinck S, Jansen R, Rijkenberg S, Wester JPJ, Bosman RJ, van der Meer NJM, van der Voort PHJ: The ecological effects of selective decontamination of the digestive tract (SDD) on antimicrobial resistance: a 21-year longitudinal single-centre study. Critical Care 2019, 23(1):208.

23. de Smet AM, Kluytmans JA, Blok HE, Mascini EM, Benus RF, Bernards AT, Kuijper EJ, Leverstein-van Hall MA, Jansz AR, de Jongh BM et al: Selective digestive tract decontamination and selective oropharyngeal decontamination and antibiotic resistance in patients in intensive-care units: an openlabel, clustered group-randomised, crossover study. Lancet Infect Dis 2011, 11(5):372-380.

24. Daneman N, Sarwar S, Fowler RA, Cuthbertson BH: Effect of selective decontamination on antimicrobial resistance in intensive care units: a systematic review and meta-analysis. The Lancet Infectious Diseases 2013, 13(4):328-341.

25. Hammond JMJ, Potgieter PD: Long-term effects of selective decontamination on antimicrobial resistance. Read Online: Critical Care Medicine | Society of Critical Care Medicine 1995, 23(4):637645 .

\section{Tables}

Table 1. Baseline characteristics. 


\begin{tabular}{|lll|}
\hline & q.i.d. SDD & t.i.d. SDD \\
\hline Total amount of admissions & 1236 & 722 \\
\hline Total patients in cohort (\% male) & $1171(67.3 \%)$ & $690(70 \%)$ \\
\hline Admission duration in days; Median (min-max) & $11(3-118)$ & $11(3-182)$ \\
\hline Age at admission day; Mean (min-max) & $62(17-92)$ & $62(17-92)$ \\
\hline
\end{tabular}

Table 2. Proportion of ICU admission with successful decontamination of GNB within 14 days (remaining negative during the entire admission period).

\begin{tabular}{|llll|}
\hline & q.i.d. SDD (\%) & t.i.d. SDD (\%) & Total \\
\hline Failure of decontamination within 14 days & $380(30,7 \%)$ & $240(33,2 \%)$ & $620(31.7 \%)$ \\
\hline Succesful decontamination within 14 days & $856(69,3 \%)$ & $482(66,8 \%)$ & $1338(68,3 \%)$ \\
\hline Total & & & \\
\hline
\end{tabular}

Table 3. Causative pathogens in intensive care unit-acquired bacteremia with GNB.

\begin{tabular}{|llll|}
\hline & q.i.d. SDD n & t.i.d. SDD n & Total n (\%) \\
\hline Pseudomonas aeruginosa & 4 & 3 & $7(26)$ \\
\hline Escherichia coli & 3 & 3 & $6(22)$ \\
\hline Enterobacter species & 3 & 0 & $3(11)$ \\
\hline Klebsiella species & 2 & 0 & $2(7)$ \\
\hline Stenotrophomonas maltophilia & 1 & 1 & $2(7)$ \\
\hline Polymicrobial & 1 & 1 & $2(7)$ \\
\hline Acinetobacter species & 0 & 1 & $1(4)$ \\
\hline Moraxella osloensis & 1 & 0 & $1(4)$ \\
\hline Ochrobactrum anthropi & 1 & 0 & $1(4)$ \\
Burkholderia cepacia & 0 & 1 & $1(4)$ \\
\hline Total & 17 & 10 & $27(100)$ \\
\hline
\end{tabular}

Table 4. Susceptibility for tobramycin or colistin in Gram-negative bacteria in baseline surveillance cultures. 


\begin{tabular}{|lll|}
\hline & $\begin{array}{l}\text { Admissions } \\
\text { during q.i.d. SDD } \\
\text { application period } \\
\mathrm{n}(\%)\end{array}$ & $\begin{array}{l}\text { Admissions } \\
\text { during t.i.d. SDD } \\
\text { application period } \\
\mathrm{n}(\%)\end{array}$ \\
\hline Total & 1236 & 722 \\
\hline $\begin{array}{l}\text { Gram-negative bacteria in baseline }(<72 \mathrm{H}) \text { surveillance } \\
\text { cultures }\end{array}$ & $880(71.2)$ & $518(71.7)$ \\
$\begin{array}{l}\text { Susceptibility for at least one of the non-absorbable } \\
\text { antibiotics in SDD (tobramycin or colistin) in Gram- } \\
\text { negative bacteria in baseline }(<72 \mathrm{H}) \text { surveillance cultures }\end{array}$ & $852 / 880(96.8)$ & $504 / 518(97.3)$ \\
\hline
\end{tabular}

\section{Figures}

\section{Detection of GNB in surveillance cultures} during admission on the ICU

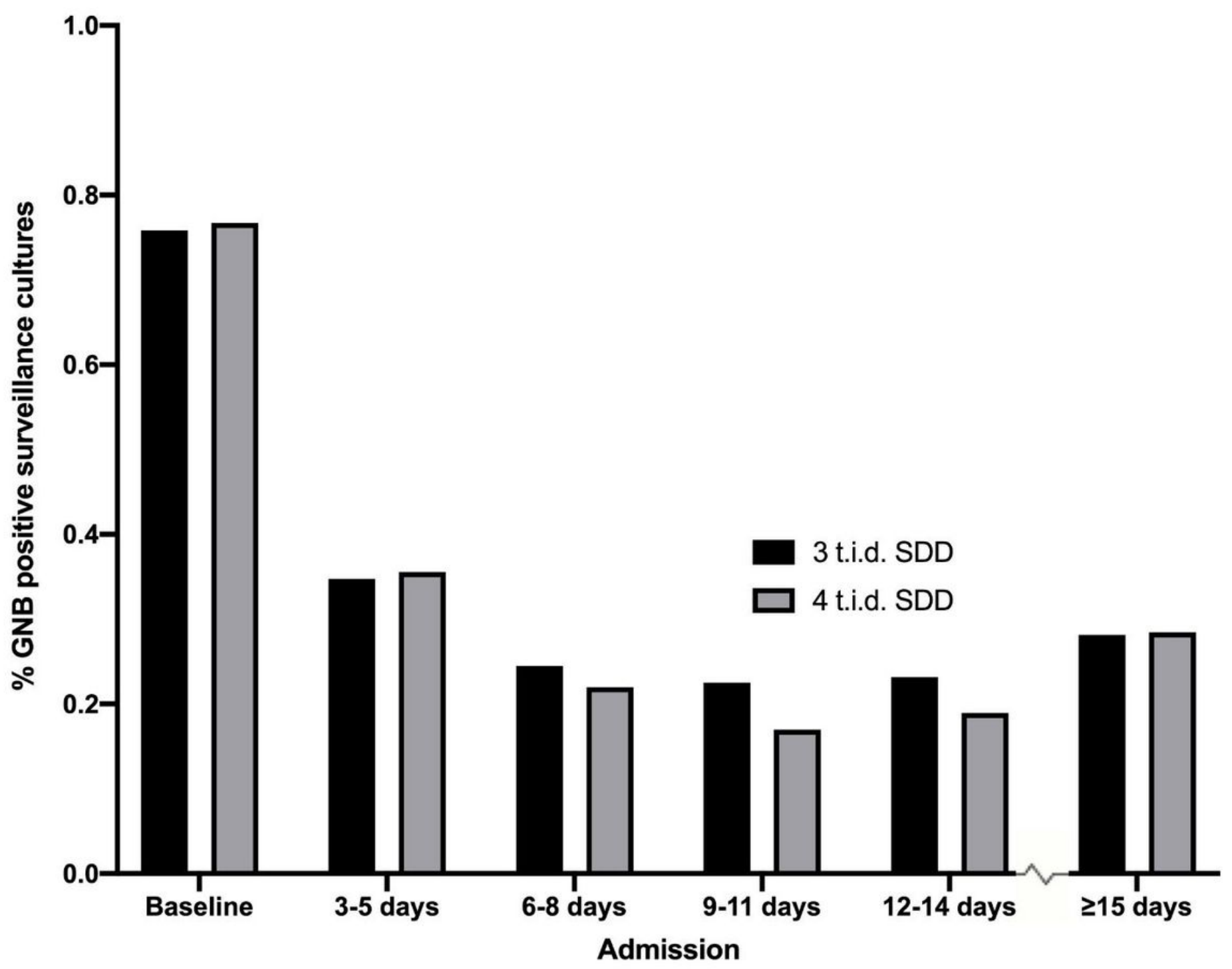

Figure 1 
Percentages of patients with Gram-negative bacteria in surveillance cultures, out of all patients with surveillance cultures drawn during admission days, are presented.

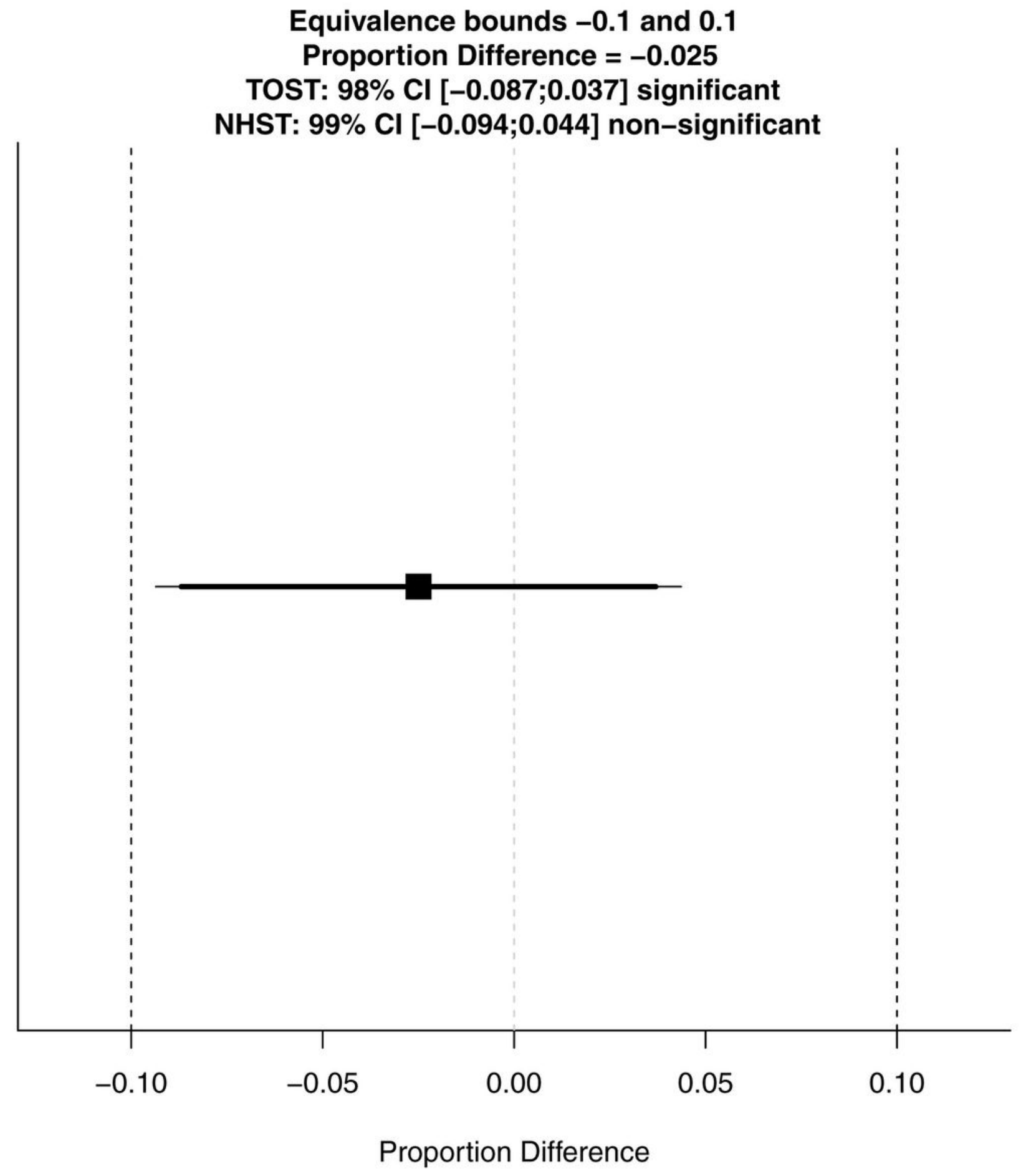

Figure 2

Equivalence test of proportion of successful decontamination 


\section{Kaplan-Meier Curves \\ Time to decontamination of GNB}

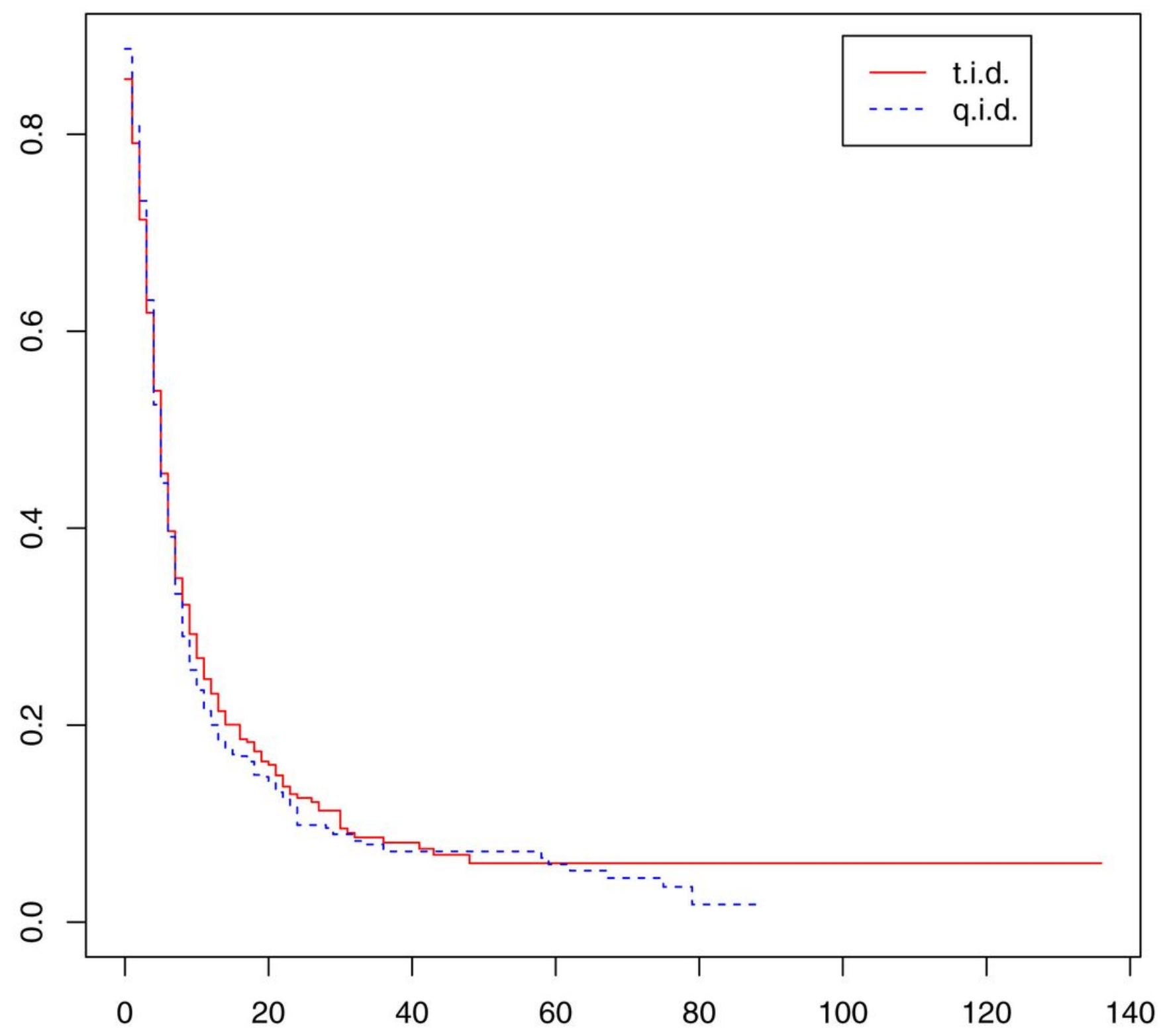

Figure 3

Time to decontamination of GNB. ( Log - rank p-value $=0.55)$ 